CBN 97-5

\title{
The HOM Study of CESR RF Cavities Using Single Circulating Bunch ${ }^{1}$
}

\author{
A. Temnykh ${ }^{2}$, D. Hartill, S. Belomestnykh and R. Kaplan \\ Laboratory of Nuclear Studies \\ Cornell University, Ithaca NY 14953, USA
}

January 28, 1997

\begin{abstract}
The CESR RF system consists of four 5-cell RF cavities [1]. Each cell has a field probe sensitive to the voltage in the cell. We used a single bunch circulating in the ring as the driving current which gives a well known spectrum. The signals from the field probes were analyzed and we were able to identify the peaks seen in the spectra as higher-order modes (HOMs) that are excited by the beam in RF cavities. Analysis of the peak shapes and location gave us the $Q$ factors of parasitic modes and their frequencies. The reliability of data was verified with direct measurement using a Network analyzer. The information about $Q$ s and frequencies of the parasitic modes will be used for the multi-bunch beam stability analysis.
\end{abstract}

\section{Introduction}

It was found that the maximum beam current at CESR is limited by the muti-bunch beam instability. The appearance of this instability is seen as the self-excited lines in the spectra of the signals picked up from BPM buttons. To determine which of CESR elements are responsible for that we performed the following experiment. Keeping beam current constant we changed the

\footnotetext{
${ }^{1}$ Work supported by the National Science Foundation

${ }^{2}$ On leave from BINP, Novosibirsk
} 


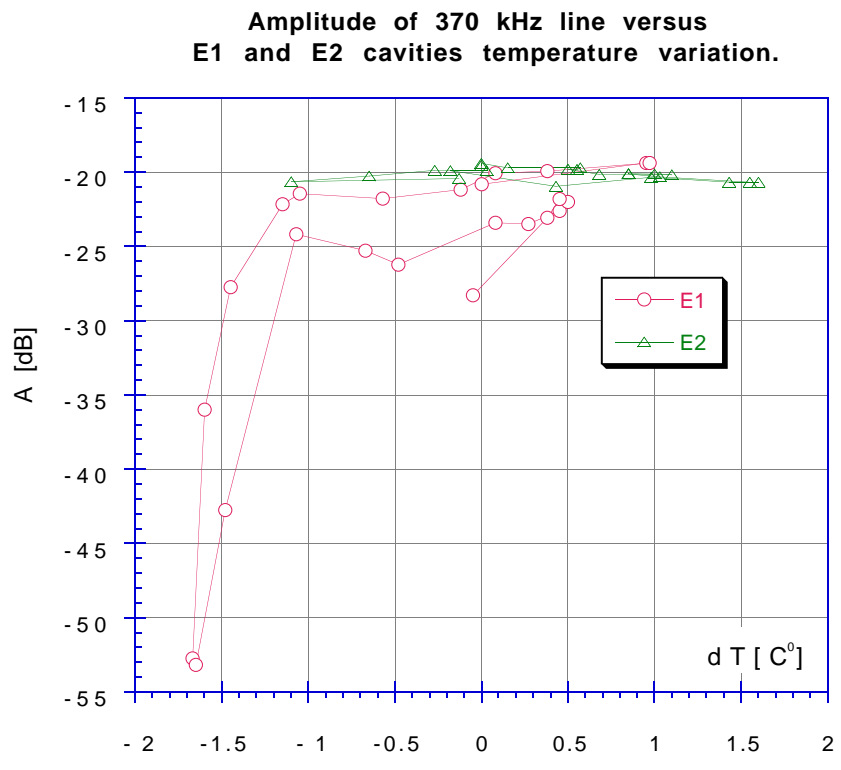

Figure 1: Amplitude of $370 \mathrm{kHz}$ line versus East RF cavities temperature.

temperature of one of the CESR RF cavities at a time and observed the amplitude of the self-excited line in the spectrum (see [2]). Results of this experiment are plotted in figures 1 and 2 .

Here the horizontal axis is the temperature of the cavities relative to operation point. Vertical axis is the amplitude of self-exited line at $370 \mathrm{kHz}$. One can see that amplitude of line is changing from $-55 \mathrm{~dB}$, which is the noise level, to $-20 \mathrm{~dB}$ level indicating strong instability. During this experiment the synchrotron frequency change was negligible. The cavity temperature variation causes the small profile distortion leading to the frequency shift of the cavity HOMs. The frequency of the fundamental mode is kept in place by the tuner, but the frequencies of all other modes can be shifted unpredictably. If the frequency of one of those HOMs will appear in the vicinity of the lines presented in the spectrum of the beam current, it may result in high parasitic voltage in cavity which may drive the beam instability. The mode voltage is proportional to the beam current and depends on the quality factor $Q$ and the mode specific impedance $R / Q$. While $R / Q$ for each mode can be calculated, the reliable value of $Q \mathrm{~s}$ and frequencies can be obtained only from 


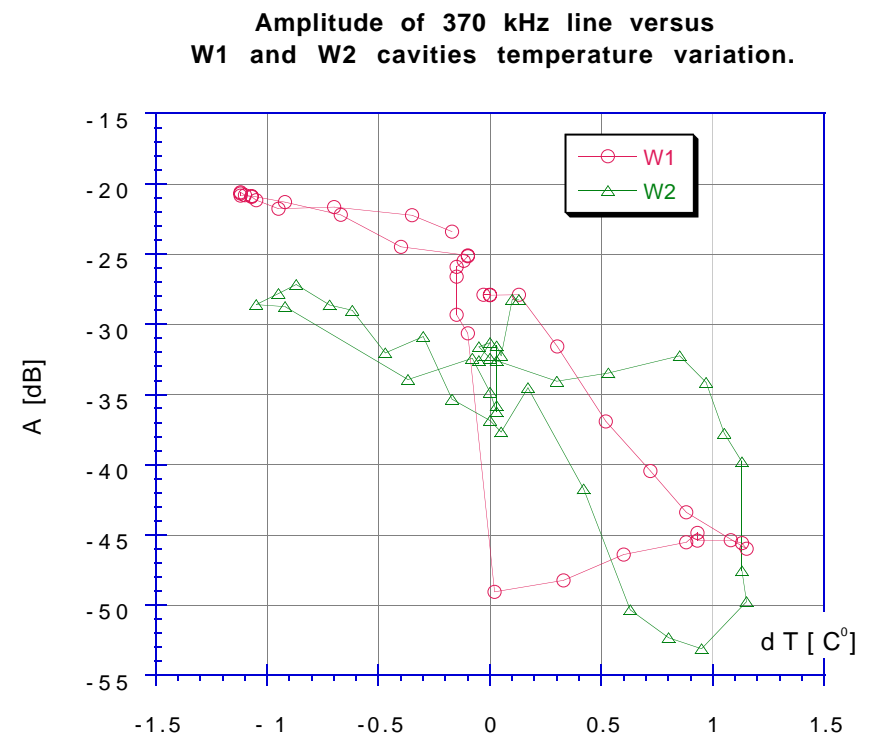

Figure 2: Amplitude of $370 \mathrm{kHz}$ line versus West RF cavities temperature.

measurements. To obtain this information for the cavities presently installed in the ring we directed experiments described below. Obtained data will help us to analyze multi-bunch beam instability and make a proper choice for the beam current increase strategy.

\section{Higher Order Modes Identification}

The CESR RF system consists of four 5-cell RF cavities. Two of them, E1 and E2, are located in the east side of the ring and two others, W1 and W2, are in the west side. Each cell has a field probe sensitive to the voltage in the cell. We analyzed the signals from these probes in our experiments. To excite higher-order modes we used a single bunch circulating in the ring which gives us simple spectrum of driving current. This spectrum contains all harmonics of the revolution frequency, $f_{0}=390.13 k H z$. The harmonic strength depends on its frequency, $f$, as $\exp \left(-f^{2} /\left(2 f_{c}^{2}\right)\right)$, where $f_{c} \sim 3 G H z$ is determined by the bunch length. Figure 3 shows spectra of a signal picked 


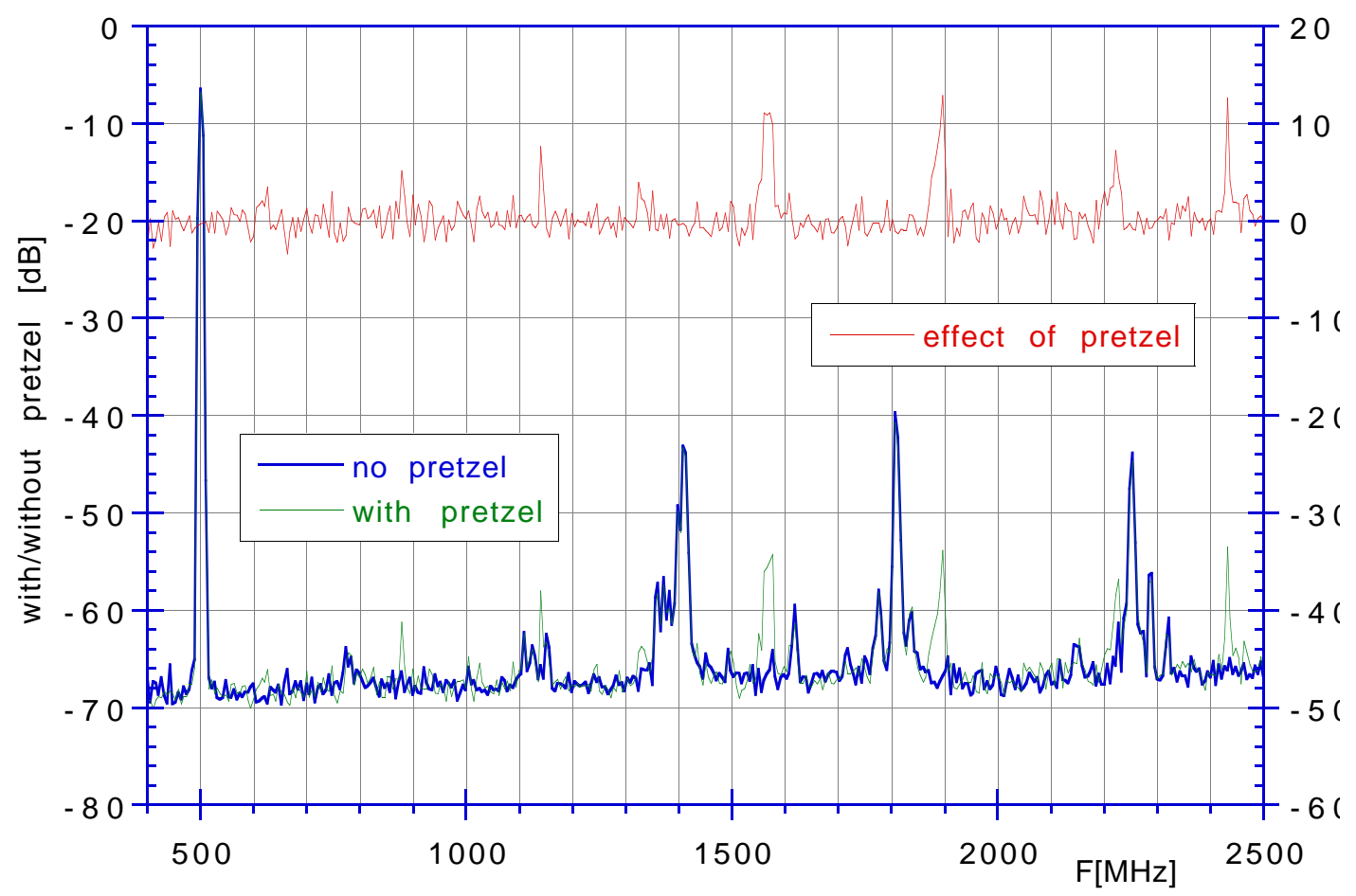

Figure 3: Spectra of signal from field probe of E1 cavity cell 4.

from the field probe of the E1 cavity cell number 4 . The circulating current was $6 \mathrm{~mA}$ in a single positron bunch. The first plot, marked as

"no pretzel", is the spectra obtained with the beam orbit close to the cavity axis. We can expect excitation of only longitudinal HOMs in this case. The second spectrum, labeled as "with pretzel", is for the pretzelized orbit, i.e. the beam orbit was shifted by approximately $10 \mathrm{~mm}$ off the axis in horizontal plane. One can see that additional peaks appear which may be interpreted as the excitation of the dipole modes. The bottom plot, "effect of pretzel", is the result of subtraction of the first spectrum from the second one.

The next step was to identify peaks seen in spectrums with calculated higher-order modes. The URMEL calculations were done for the single-cell. Table 1 shows the calculated characteristics of longitudinal modes found by URMEL in the frequency range from $500 \mathrm{MHz}$ to $2.5 \mathrm{GHz}$. It contains 


\begin{tabular}{|c|c|c|c|c|}
\hline \hline Number & Type & $\mathrm{f}[\mathrm{MHz}]$ & $R / Q[\Omega]$ & seen in spectra \\
\hline 1 & TM0-Ex-1 & 498.16 & 132.47 & yes \\
2 & TM0-Mx-1 & 773.52 & 25.645 & yes \\
3 & TM0-Ex-2 & 1125.4 & 1.6010 & yes \\
4 & TM0-Ex-3 & 1351.4 & 6.0730 & yes \\
5 & TM0-Mx-2 & 1401.8 & 12.578 & yes \\
6 & TM0-Ex-4 & 1750.5 & 5.0770 & yes \\
7 & TM0-Mx-3 & 1791.5 & 4.9880 & yes \\
8 & TM0-Ex-5 & 1961.0 & 0.0170 & no \\
9 & TM0-Mx-4 & 2146.0 & 0.5930 & $?$ \\
10 & TM0-Ex-6 & 2228.7 & 3.4640 & yes \\
11 & TM0-Mx-5 & 2274.2 & 1.2160 & yes \\
\hline \hline
\end{tabular}

Table 1: List of longitudinal modes found by URMEL between $500 \mathrm{MHz}$ and $2.5 \mathrm{GHz}$.

frequencies and $R / Q \mathrm{~s}$. The measured spectrum for the on axis beam orbit is shown in figure 4. Dots represent calculated HOMs. We used the logarithm of the ratio of a mode $R / Q$ to the fundamental mode $R / Q$ for the vertical positioning the dots. It gives us a sense of the relative mode strength.

There is no doubt that the peaks in the spectra are related to the higherorder modes and we can say with certain which mode is responsible for the appearance of which peak. Note that the field probe sensitivity is different for different modes. It depends on the probe location and on the mode field pattern. The mode with the higher voltage may give the peak with smaller amplitude than the other one with the lower voltage. So, it would be wrong to compare measured peaks amplitude with calculated $R / Q$.

The same procedure was done for the dipole modes. Figure 5 shows the difference between two spectra, with and without pretzel. Here, also, one can see correlation between peaks and dots representing calculated HOMs, (see Table 2). So we again are able to say which peak corresponds to which mode.

The next step was to study the characteristics of higher-order modes needed for multi-bunch beam stability analysis. 


\begin{tabular}{|c|c|c|c|c|}
\hline Num & Type & $\overline{\mathrm{f}[\mathrm{MHz}]}$ & $\bar{R} R / Q @ 4.4 \mathrm{~cm}[\Omega]$ & " seen in spectrum \\
\hline 1 & $1-\mathrm{Mx}-1$ & 747.19 & 0.77700 & \\
\hline 2 & 1-Ex-1 & 871.35 & 17.696 & $\mathrm{X}$ \\
\hline 3 & $1-\mathrm{Mx}-2$ & 1138.8 & 24.971 & $\mathrm{X}$ \\
\hline 4 & 1-Ex-2 & 1225.8 & 0.014000 & \\
\hline 5 & $1-\mathrm{Mx}-3$ & 1327.6 & 0.91400 & $\mathrm{X}$ \\
\hline 6 & 1-Ex-3 & 1500.5 & 0.87600 & \\
\hline 7 & $1-\mathrm{Mx}-4$ & 1562.2 & 19.187 & $\mathrm{X}$ \\
\hline 8 & 1-Ex-4 & 1621.8 & 0.57700 & \\
\hline 9 & 1-Ex-5 & 1749.8 & 0.18100 & \\
\hline 10 & $1-\mathrm{Mx}-5$ & 1879.8 & 6.5990 & $\mathrm{X}$ \\
\hline 11 & $1-\mathrm{Ex}-6$ & 1885.6 & 19.667 & $\mathrm{X}$ \\
\hline 12 & $1-\mathrm{Mx}-6$ & 1948.8 & 0.061000 & \\
\hline 13 & $1-\mathrm{Mx}-7$ & 2046.4 & 0.91400 & \\
\hline 14 & 1-Ex-7 & 2196.6 & 3.0320 & $\mathrm{X}$ \\
\hline 15 & $1-M x-9$ & 2223.9 & 2.7300 & $\mathrm{X}$ \\
\hline 16 & 1-Ex-8 & 2264.4 & 0.63000 & \\
\hline 17 & 1-Ex-9 & 2354.1 & 2.1100 & \\
\hline 18 & 1-Ex-10 & 2491.0 & 1.7360 & \\
\hline
\end{tabular}

Table 2: List of dipol modes found by URMEL between $500 \mathrm{MHz}$ and 2.5 GHz. 


\section{Longitudinal mode identification.}

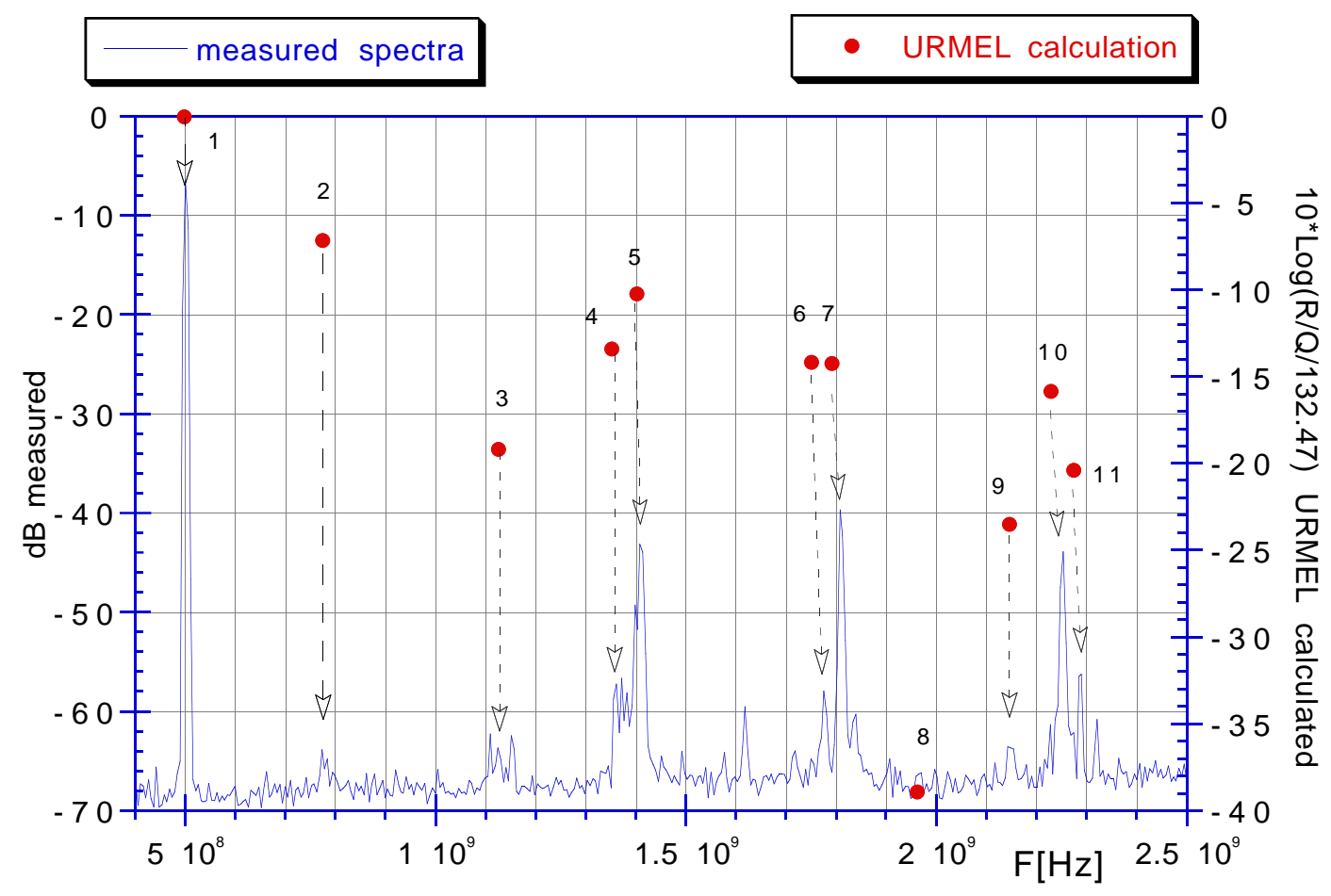

Figure 4: Longitudinal modes identification.

\section{HOM characteristics study}

Figure 5 shows a spectrum of signal from the E1 cell 4 field probe in a $\pm 3.5 \mathrm{MHz}$ frequency range around $1801.5 \mathrm{MHz}$. We know from the previous measurements that here is a peak caused by the TM0-Mx-3 mode excitation. The form of the spectrum seen in figure 6 reflects the spectrum of driving current as well as shape of the resonance. As far as we used a single circulating bunch, its spectrum has lines at harmonics of the revolution frequency. The envelope of the spectrum is determined by the TM0-Mx-3 mode parameters. This envelope was fitted with the theoretical resonant function using the resonant frequency $f_{m}$ and the quality factor $Q$ as free parameters. The 


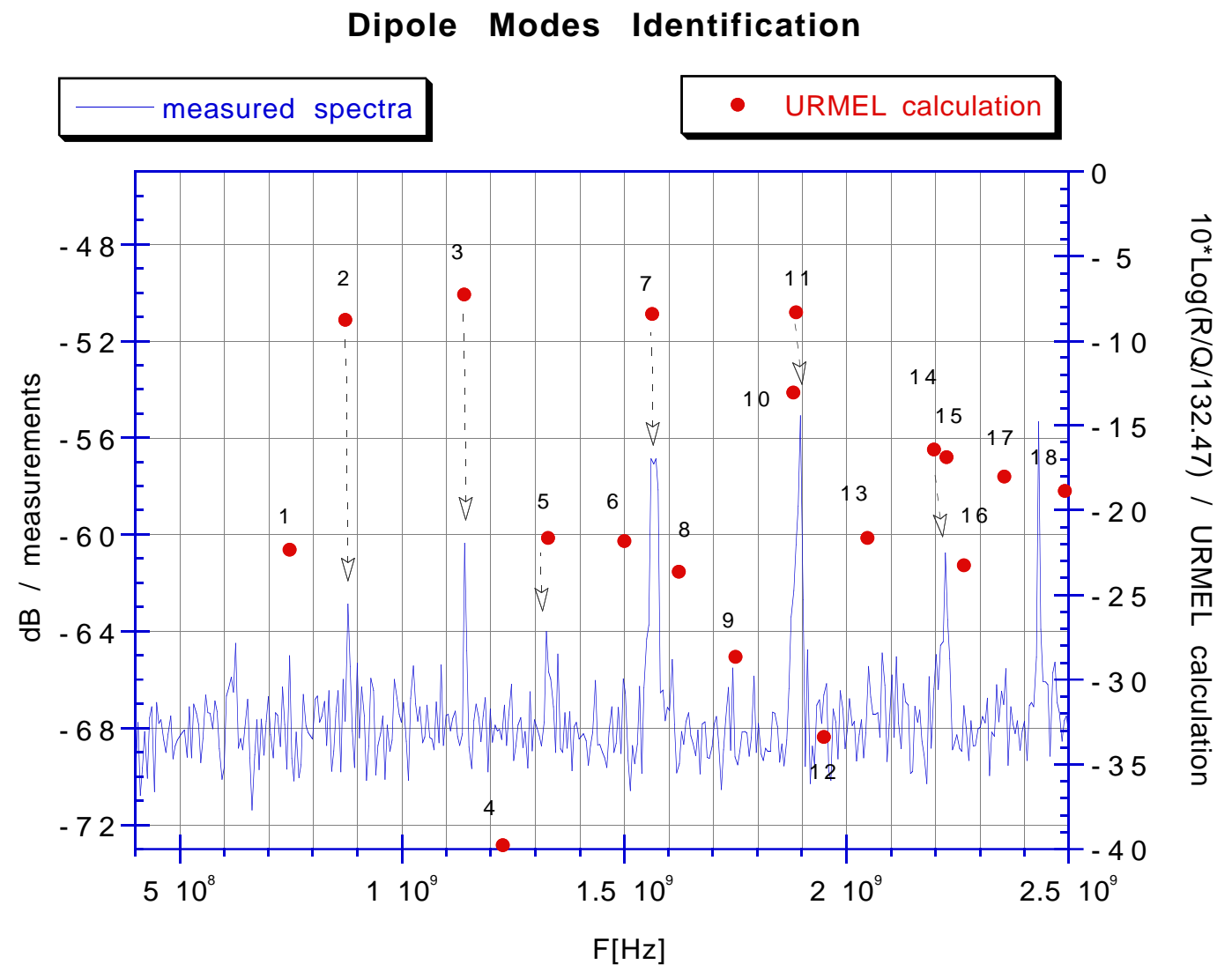

Figure 5: Dipol modes identification.

best fitting was obtained with $Q=15,853 \pm 9,000$ and $f_{m}=1801.4 \mathrm{MHz}$. One can see relatively large uncertainty in Q. It occurs because the fitted points are sampled by the revolution frequency and in the case of high $Q$ only few of them, which are close to the mode frequency, play role in the $Q$ determination. In the case of low $Q$ the number of points important for the $Q$ determination is getting larger and the accuracy becomes better. The accuracy of the mode frequency fitting is always better than $0.1 \mathrm{MHz}$.

To verify these numbers we performed the direct measurements of the cell 4 of the E1 cavity using a Network analyzer. The higher-order mode probe [3] was used to drive field in the cell and field probe was used to pick up the signal. By measuring the S21 function we found the peak of the TM0- 
Resonance fitting of the measured single bunch spectra around $1800 \mathrm{MHz}$.

East RF cavity 1 , cell 4 .

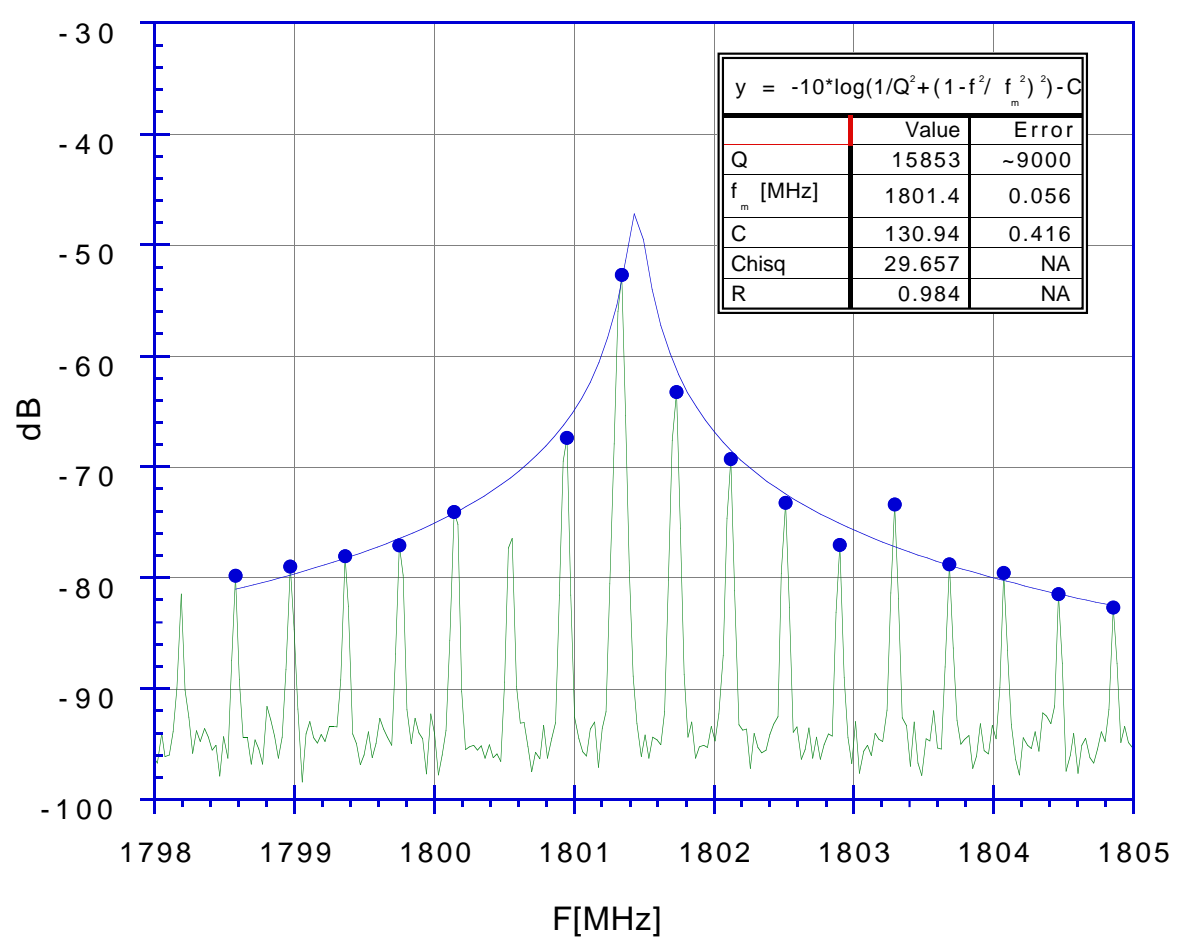

Figure 6: Resonance fitting of spectrum around TM0-ME-3 mode location. E1 cavity, cell 4.

Mx-3 mode with $Q=16,400$ and $f_{m}=1801.563 \mathrm{MHz}$. These numbers are in a good agreement with numbers obtained from the beam measurement. The CESR RF system contains a total of 20 accelerating cells. All of them have slightly different spectra, i.e. slightly different peak amplitudes and positions. It may be easily explained by the their mechanical differences. For the purpose of the muti-bunch beam stability analysis it is necessary to know the mode frequency and $Q$ variation among the cells. Table 3 shows the result of analysis of two longitudinal modes, TM0-Mx-2 and TM0-Mx-3, for 12 cells. One can see that the TM0-Mx-3 mode has average frequency 


\begin{tabular}{|c|c|c|c|c|}
\hline \hline Cavity/cell & \multicolumn{2}{|c|}{ TM0-Mx-2 mode } & \multicolumn{2}{c|}{ TM0-Mx-3 mode } \\
\hline & $f_{m}[M H z]$ & $Q$ & $f_{m}[M H z]$ & $Q$ \\
\hline E1/1 & 1405.3 & 485 & 1800.4 & 12300 \\
E1/2 & 1409 & 507 & 1803.7 & 8922 \\
E1/4 & 1404.9 & 1007 & 1801.4 & 24800 \\
E1/5 & 1405.7 & 3168 & 1803 & 5302 \\
E2/1 & 1404 & 506 & 1802 & 12484 \\
E2/2 & 1410.2 & 2117.6 & 1806.5 & 5548 \\
W1/1 & 1404.1 & 614 & 1801.3 & 9227 \\
W1/2 & & & 1804.9 & 3535 \\
W1/4 & 1406.1 & 613 & 1803.4 & 5736 \\
W1/5 & 1407.3 & 1944 & 1804.2 & 3860 \\
W2/1 & 1406.9 & 422 & 1803.8 & 18842 \\
W2/2 & 1407 & 550 & 1803.5 & 20000 \\
\hline Average & 1406.41 & 1085 & 1803.18 & 10879.67 \\
RMS & 1.94 & 914 & 1.70 & 7011.19 \\
\hline \hline
\end{tabular}

Table 3: Characteristics of the TM0-Mx-2 and TM0-Mx-3 modes.

of $1803.2 \mathrm{MHz}$ with $\pm 1.8 \mathrm{MHz}$ rms. spread among the cells. Its average $Q$ is about 11,000 with $\pm 7,000 \mathrm{rms}$. The TM0-Mx-2 mode characteristics are $1406.41 \pm 1.94 \mathrm{MHz}$ frequency and $Q$ in a range from 400 to 3,000. Note that the TM0-Mx-2 modes have remarkably lower $Q \mathrm{~s}$ than those of TM0-Mx-3.

As an example of variation of the mode parameters we show the spectra around the central frequency of $1802 \mathrm{MHz}$ for 4 cells of the E1 cavity (figure 7 ). One can definitely see the spread of the mode frequency as well as variation in the shapes of fitting curves reflecting different $Q \mathrm{~s}$.

\section{Conclusion}

Peaks seen in spectra of the signals from the RF field probes have been identified as higher-order modes exited by the beams. We measured Q factors and tunes of the TM0-Mx-2 and TM0-Mx-3 modes in 12 RF cells using a single circulating bunch as a driving current . This information will be used for multi-bunch beam stability analysis. 


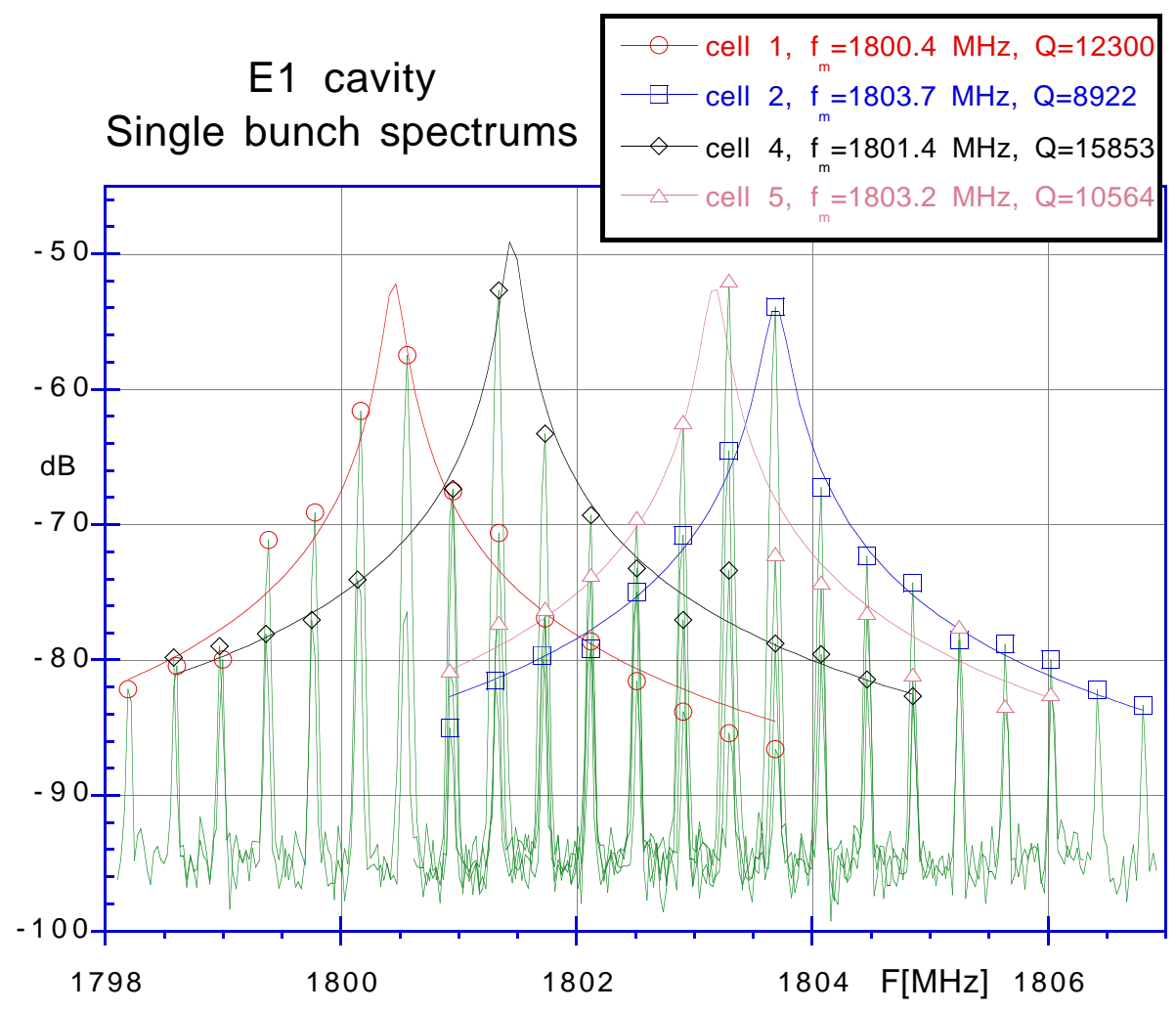

Figure 7: Spectra of the signals from four cells of the E1 cavity showing TM0-Mx-3 mode excitation

\section{Acknowledgment}

We wish to thank all people of the CESR operating group who assisted us in our measurements.

\section{References}

[1] RF Cavity Design for High Current Operation of the Cornell Electron Storage Ring, S. Greenwald, et al. Proceedings of the 1989 Particle Accelerator Conference, Chicago, IL, March 1989, Vol. 1, pp. 226-228 
[2] CESR Elog: Machine Studie, CESR Impedance and Instabilities, Sept 171996

[3] CESR RF System, R. Sundelin, et al. IEEE Trans. on Nucl. Sci., Vol. NS-28, No. 3, Part 2 , pp. 2844-2846 (June 1981). 